\title{
Spectrum Availability Recheck Scheme (SARS) for Cognitive Radio Systems with Mobility
}

\author{
Woongsup Lee* and Dong-Ho Cho ${ }^{\dagger}$ \\ Department of Electrical Engineering \\ Korea Advanced Institute of Science and Technology (KAIST) \\ Daejeon, 305-701, Republic of Korea \\ E-mail : ${ }^{*}$ wslee@ comis.kaist.ac.kr, ${ }^{\dagger}$ dhcho@ee.kaist.ac.kr
}

\begin{abstract}
We consider the change of spectrum availability which is caused by the mobility of a cognitive radio (CR) user. Given that the CR user should not interfere with a primary user (PU), it should periodically check the availability of the CR band which is being used. Herein, we refer the scheme to check the availability of spectrum as SARS (Spectrum Availability Recheck Scheme). We propose a SARS for both CR systems which use a geo-location database to find vacant band and CR systems which use spectrum sensing to find vacant band. The proposed SARS adaptively changes its operation according to the velocity of a CR user. Through the analysis and the simulation results, we have shown that the spectrum availability of a CR user can be efficiently verified by using proposed SARS, when the CR user moves. We also have shown that the overheads caused by our SARS is low.
\end{abstract}

\section{INTRODUCTION}

In these days, the amount of mobile data traffic has been explosively increasing. To support this increase in traffic, cognitive radio (CR) technology has been introduced [1] [2]. In the CR technology, the wireless system finds vacant band which is not being used by a primary user (PU) who has the right to the band, and uses those bands unless their use interrupts the PU. Therefore, the detection of band which is vacant is a critical factor for $\mathrm{CR}$ systems.

From previous researches, two schemes to find vacant band have been considered, which are geo-location database based scheme (DB based scheme) and spectrum sensing based scheme (sensing based scheme) [3]. In the DB based scheme, a CR system finds vacant bands by accessing the database which contains the location information of PU systems and looking into the spectrum usage at its location [3]. And in the sensing based scheme, the status of the bands can be found by using the spectrum sensing. In this scheme, each CR user should perform spectrum sensing to find a vacant band [10].

After vacant bands have been found, the CR system can use the bands and it should periodically check whether the band which is being used, is still vacant. Herein, we refer the method to check the spectrum availability as SARS (Spectrum Availability Recheck Scheme). In the CR environments, the spectrum availability of a CR user can change due to two reasons, which are 1) the temporal variation of PU activity and 2) the spatial movement of a CR user [4] [7]. In previous researches, a SARS for the temporal variation of PU activity has been extensively studied [4] [5]. However, a SARS for the spatial movement of a CR user has not been studied previously.

Herein, we consider a SARS which considers the mobility of a CR user in a DB based scheme and a sensing based scheme. For the SARS in the DB based scheme, we consider two schemes which are a GPS assisted SARS and a spectrum sensing assisted SARS. In the GPS assisted SARS, the period of location update of the CR user adaptively changes according to the velocity of the CR user. On the other hand, in the spectrum sensing assisted SARS, an additional spectrum sensing is used to reduce the number of the location update, because the location update requires high energy consumption and computations. For the SARS in the sensing based scheme, we consider an adaptive sensing period scheme in which the period of spectrum sensing is changed according to the velocity of the CR user. By using our proposed SARS, the spectrum availability can be efficiently checked when the CR users has mobility.

\section{Problem StATEMEnT}

When a CR user moves, the spectrum availability of the CR user will change accordingly. Fig.1 shows the change of the spectrum availability of the CR user. In Fig.1, the no-talk zone of a PU is the protected area of the PU in which the CR user should not use the band [10]. As we can see from the figure, a CR band is initially available for both CR user 1 and CR user 2, however, the CR band becomes unavailable when $t=3$ and $t=1$, respectively. If the temporal variation of the PU activity is static such as the case of DTV station [7] [9], the change of spectrum availability due to the mobility of the CR user will be more significant compared to that due to the temporal variation of the PU activity. The impact of the mobility becomes greater when the velocity of a CR user is high as we can see from Fig.1. Therefore, a SARS for the spatial movement of a CR user is critical and proper solution should be devised. The SARS with this type has not been studied in previous researches. In [7], the authors considered the change of spectrum availability which is caused by the mobility of a CR user. However, they did not propose a method to deal with this change of spectrum availability.

Herein, we consider a SARS which concerns the mobility of a CR user. We propose a SARS for both a DB based 


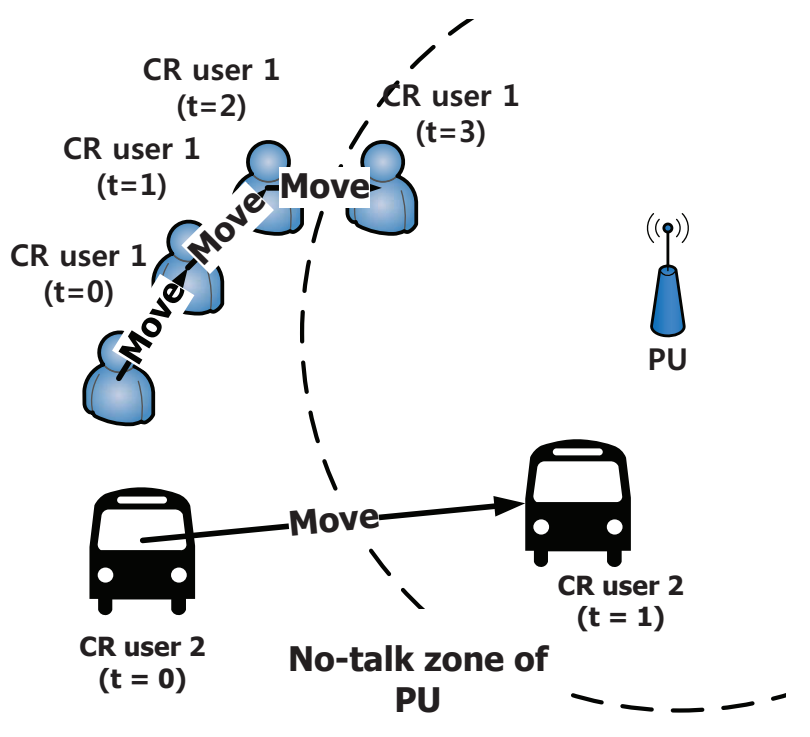

Fig. 1. Change of spectrum availability due to $\mathrm{CR}$ user mobility

scheme and a sensing based scheme. Our SARS can be used for the uplink channel for a cellular CR systems or adhoc CR systems. For the downlink channel of the cellular CR systems, the SARS which considers the mobility of a $\mathrm{CR}$ user is unnecessary because a CR base station cannot move. Moreover, the SARS should change its operation adaptively according to the velocity of $\mathrm{CR}$ users, because when a CR user moves fast, the spectrum availability will change more frequently. In the next two chapters, we will explain the SARS that we have proposed.

\section{SARS IN DB BASED SCHEME}

We first consider a SARS in a DB based scheme. In this case, we assume that a CR user can acquire its location information by using GPS because without the location information of the CR user, the DB based scheme is hard to be used $[7]^{1}$. For the SARS in the DB based scheme, we consider two schemes, which are a GPS assisted SARS and a spectrum sensing assisted SARS.

In a GPS assisted SARS, a CR user periodically finds its position by using GPS and checks whether it is within the notalk zone of the PU. The CR user should not use the band if it is within the no-talk zone of the PU. Therefore, if the CR user is within the no-talk zone, it should use other CR band. In the GPS assisted SARS, the period of location update, which we denote as $\Delta t^{G P S}$ should be carefully chosen, because if the period is too short, the power consumption and computational overhead due to the use of GPS will increase and if the period is too long, the PU can be interfered by the CR user.

To derive proper $\Delta t^{G P S}$, we assume that the maximum velocity of a CR user is $v_{\max }$. And we also assume that

\footnotetext{
${ }^{1}$ As we can see from [7], the CR user in a cellular system may use the band which is available for all the coverage of the base station. In this case, the CR user should change the band when it moves to the coverage of other base station.
}

the distance between the PU and the CR user is $D_{\min }^{P U}$. By considering the worst case in which the $\mathrm{CR}$ user moves toward the PU with maximum velocity, $\Delta t^{G P S}$ can be calculated as follows ${ }^{2}$ :

$$
\Delta t^{G P S}=\min \left(\frac{D_{\min }^{P U}}{v_{\max }}, t_{\min }^{G P S_{-} u p d a t e}\right)
$$

where $t_{\text {min }}^{G P S \_ \text {update }}$ is the minimum duration between two consecutive GPS readings ${ }^{3}$.

However, a GPS assisted SARS has the problem of frequent GPS usage. In the GPS assisted SARS, a CR user should use a GPS to find its location in every $\Delta t^{G P S}$ period. Given that the power consumption and the computational overhead which are caused by a GPS reading are high, it is preferable to reduce the amount of GPS reading as small as possible.

To decrease the number of GPS readings, we propose a spectrum sensing assisted SARS which utilizes spectrum sensing. In the spectrum sensing assisted SARS, a CR user periodically measures the received signal power on the PU which does not use the same band that the CR user is using. By measuring the received signal power from the active PU which is in proper location, the CR user can estimate the distance between the PU and itself. Herein, we refer this PU as a test PU and the PU which uses the same band as the CR user as victim PU.

In the spectrum sensing assisted SARS, the test PU is selected based on certain criteria. The test PU should be an active PU which resides between the CR user and the victim PU. And the distance between the test PU and the CR user should be less than $d_{x}^{\text {test }}$ and the distance between the test PU and the line joining the CR user and the victim PU should be less than $d_{y}^{\text {test }}$. When multiple PUs can be the candidate for the victim PU, the PU which is closest to the line joining the $\mathrm{CR}$ user and the victim PU should be selected as the victim PU. If no PU satisfies the criteria of the test PU, the spectrum sensing assisted SARS scheme cannot used and the GPS assisted SARS scheme should be used instead. However, given that multiple CR bands will exist, the criteria of the test $\mathrm{PU}$ is likely to be satisfied.

Fig. 2 shows the selection of a test PU in the spectrum sensing SARS. As we can see from the figure, active PU 1 and active PU 2 are not chosen because they do not satisfy the geographical criteria of the test PU. Active PU 3 and active PU 4 are the candidate for the test PU and the active PU 4 is chosen because it is closer to the line joining a CR user and a victim PU.

Given that the selected test PU will be close to the line joining the $\mathrm{CR}$ user and the victim $\mathrm{PU}$, the $\mathrm{CR}$ user can estimate the distance between the victim PU and itself by measuring the received signal power of the test PU. And given that the test PU is much closer to the CR user compared to the

\footnotetext{
${ }^{2}$ If the actual velocity of the $\mathrm{CR}$, which we denote as $v_{\text {act }}$ can be known by using the velocity estimation schemes [8], eq.(1) can be rewritten as $t^{G P S}=$ $\frac{D_{\min }^{P U}}{v_{a c t}}$

${ }^{3} t_{\text {min }}^{\text {GPS }}$ ipdate in eq,(1) is necessary, because otherwise $t^{G P S}$ converges to zero.
} 


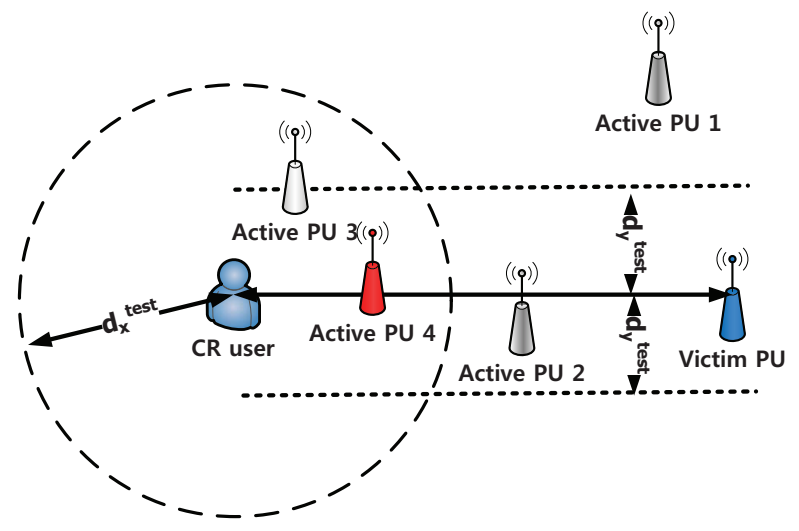

Fig. 2. Selection of a test PU in the spectrum sensing assisted SARS

victim PU, the accuracy of this distance estimation will be high even though the number of samples is not large. Therefore, the measurement can be done within very short time ${ }^{4}$ and this additional spectrum sensing will not impose large overhead to a CR systems.

To estimate the distance between a CR user and a victim $\mathrm{PU}$, the CR user first measures the received signal power of the test PU by using energy detection. The received signal from the test PU, which we denote as $y_{d_{i n i t}}(n)$, can be represented as follows [12]:

$$
y_{d_{\text {init }}}(n)=h_{n} \cdot\left(P_{T} \cdot \kappa \cdot d_{\text {init }}^{-\alpha}\right)^{\frac{1}{2}}+w_{n} \cdot(N 0 W)^{\frac{1}{2}}
$$

where $P_{T}$ is the transmission power of the test PU, $\kappa$ is the path loss constant, $\alpha$ is the path loss exponent, $N 0 W$ is the power of AWGN, and $d_{\text {init }}$ is the initial distance between the CR user and the test PU [11]. Moreover, $h_{n}$ and $w_{n}$ are the independent zero-mean circularly symmetric complex Gaussian (CSCG) random variables with variance 1 . Then, by using energy detection [12], the received signal power can be calculated as follows:

$$
T\left(y_{d_{i n i t}}\right)=\frac{1}{N} \sum_{n=1}^{N}\left|y_{d_{i n i t}}(n)\right|^{2}
$$

where $N$ is the number of samples used in the energy detection.

Then, by using the central limit theorem, the distribution of $T_{\left(y_{d_{\text {init }}}\right)}$ can be approximated by Gaussian whose mean is $\mu_{d}$ and whose variance is $\sigma_{d}^{2}$. $\mu_{d}$ and $\sigma_{d}^{2}$ can be written as follows:

$$
\begin{aligned}
\mu_{d} & =P_{T} \cdot \kappa \cdot d_{\text {init }}^{-\alpha}+N 0 W \\
\sigma_{d}^{2} & =\frac{1}{N}\left(P_{T} \cdot \kappa \cdot d_{\text {init }}^{-\alpha}+N 0 W\right)^{2}
\end{aligned}
$$

Given that we choose a PU which is close to the CR user, $P_{T} \cdot \kappa \cdot d_{\text {init }}^{-\alpha}$ is likely to be much greater than the power of ambient noise, $N 0 W$. Therefore, eq.(4) can be approximated as follows:

$$
\begin{aligned}
& \mu_{d} \approx P_{T} \cdot \kappa \cdot d_{\text {init }}^{-\alpha} \\
& \sigma_{d}^{2} \approx \frac{1}{N}\left(P_{T} \cdot \kappa \cdot d_{\text {init }}^{-\alpha}\right)^{2}
\end{aligned}
$$

\footnotetext{
${ }^{4}$ As we will see in the performance evaluation, the measurement can be done within few milli seconds, maximally.
}

Then, a CR user periodically measures the received signal power of a test PU. Let this measured signal power be $T\left(y_{d_{c u r r}}\right)$ where $d_{\text {curr }}$ is the distance between the test PU and the CR user at current position. If the CR user moves closer to the victim PU, $T\left(y_{d_{c u r r}}\right)$ will be somewhat larger than $T\left(y_{d_{i n i t}}\right)$, which is the measured signal power at the initial location. Therefore, by calculating $T\left(y_{d_{c u r r}}\right)-T\left(y_{d_{i n i t}}\right)$, the CR user can estimate how close it is to a victim PU. In a spectrum sensing assisted SARS, the CR user starts to receive GPS signal, after when $T\left(y_{d_{c u r r}}\right)-T\left(y_{d_{\text {init }}}\right)>\epsilon_{d}$, and therefore the spectrum sensing assisted SARS will operate as same as a GPS assisted SARS.

In a spectrum sensing assisted SARS, $\epsilon_{d}$ should be carefully chosen for the performance of CR systems. Herein, we chose $\epsilon_{d}$, so that the probability that the victim PU is interfered by a CR user to be less than $P_{M D}^{t h r}$. To calculate the $\epsilon_{d}$, we first calculate $T\left(y_{d_{\text {curr }}}\right)$ when the CR user is at the edge of the no-talk zone of the victim PU [10]. Let $d_{f i n}$ be the distance between the test PU and the CR user when the CR user is at the edge ${ }^{5}$. Then, $T\left(y_{d_{f i n}}\right)$ will be Gaussian random variable whose mean is $P_{T} \cdot \kappa \cdot d_{f i n}^{-\alpha}+N 0 W$ and the variance is $\frac{1}{N}\left(P_{T} \cdot \kappa \cdot d_{f i n}^{-\alpha}+N 0 W\right)^{2}$. Given that the addition of two independent Gaussian random variables is also Gaussian random variable, the distribution of $T\left(y_{d_{c u r r}}\right)-T\left(y_{d_{\text {init }}}\right)$ will be Gaussian whose mean $\mu_{\epsilon}$ and variance $\sigma_{\epsilon}^{2}$ can be approximated as follows:

$$
\begin{aligned}
& \mu_{\epsilon} \approx P_{T} \cdot \kappa \cdot\left(d_{\text {fin }}^{-\alpha}-d_{\text {init }}^{-\alpha}\right) \\
& \sigma_{\epsilon}^{2} \approx \frac{1}{N}\left(\left(P_{T} \cdot \kappa \cdot d_{\text {fin }}^{-\alpha}\right)^{2}+\left(P_{T} \cdot \kappa \cdot d_{\text {init }}^{-\alpha}\right)^{2}\right)
\end{aligned}
$$

Then, $\epsilon_{d}$ which makes the probability that the victim PU is interfered by a CR user to be less than $P_{M D}^{t h r}$, can be calculates as follows:

$$
\epsilon_{d}=\mu_{\epsilon}+\sqrt{2} \cdot \sigma_{\epsilon} \cdot \operatorname{erfc}^{-1}\left(2 \cdot\left(1-P_{M D}^{t h r}\right)\right)
$$

where $\operatorname{erfc}^{-1}(x)$ is the inverse complementary error function. The procedure of a spectrum sensing assisted SARS is shown in Fig.3.

\section{SARS IN SENSING BASED SCHEME}

Now, we will consider a SARS in a sensing based scheme. In the sensing based scheme, a CR user should periodically perform spectrum sensing on the band that it is using. Given that the band is periodically checked, the change of spectrum availability due to the mobility of the CR user can be properly handled. However, given that the period of spectrum sensing can be long, a PU can be interfered by the CR user between two consecutive spectrum sensing attempts ${ }^{6}$. And it is also not desirable to reduce the period of sensing as short as possible because when the period is too short, the time spent on specturm sensing will be large and the performance of the CR systems will severely deteriorate [12].

\footnotetext{
${ }^{5}$ Given that the no-talk zone of the victim PU will be circular shape, there will be multiple candidate values for $d_{f i n}$. In this paper, we select the edge point which is closest to the initial position of the CR user.

${ }^{6}$ The period of spectrum sensing is 30 seconds in the IEEE 802.22 system [9], and the PU can be severely interfered by the CR user during this period.
} 


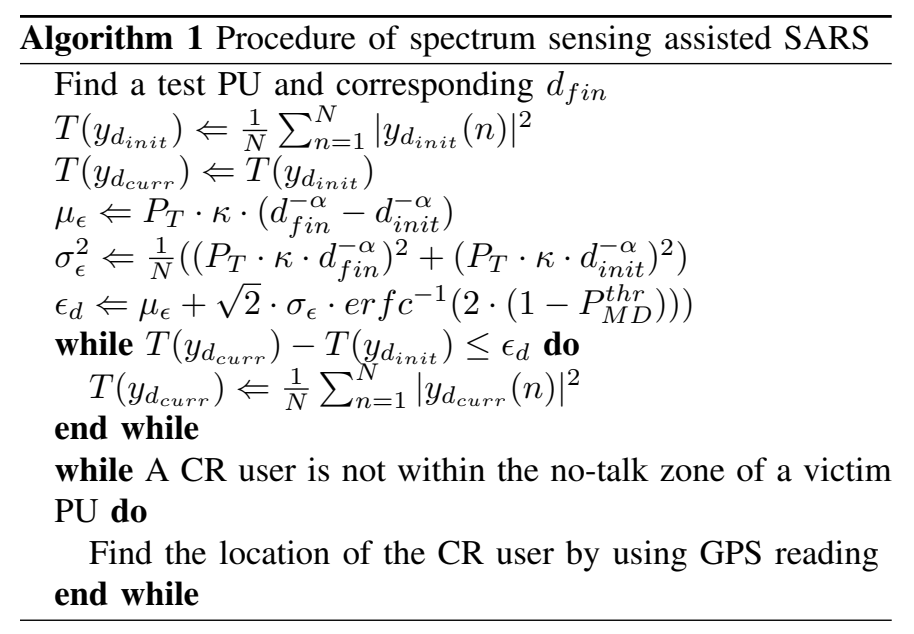

Fig. 3. Procedure of spectrum sensing assisted SARS

To solve the problem in a sensing based scheme, we propose an adaptive sensing period scheme in which the period of spectrum sensing is adaptively changed according to the velocity of a CR user. Let $t_{\text {sen }}^{\max }$ and $t_{\text {sen }}^{\min }$ be the maximum and the minimum value of sensing period, respectively, and $v_{\max }$ be the maximum velocity of the CR user. And let $R_{n o t a l k}$ be the radius of the no-talk zone of a PU. Then, in the adaptive sensing period scheme, the CR user increases the radius of the no-talk zone to $R_{\text {no_talk }}^{r e v}$ which can be written as follows:

$$
R_{n o_{-} \text {talk }}^{\text {rev }}=R_{\text {no_talk }}+v_{\max } \cdot t_{\text {sen }}^{\max }
$$

Given that the radius of the no-talk zone is increased, the sensing threshold will be decreased accordingly. The revised no-talk zone is selected so that the CR user cannot enter the original no-talk zone of the PU within one sensing period if the $\mathrm{CR}$ user is not within the revised no-talk zone. Before the $\mathrm{CR}$ user enters the revised no-talk zone, the $\mathrm{CR}$ user performs spectrum sensing with period of $t_{\text {sen }}^{\max }$. And when the $\mathrm{CR}$ user enters the revised no-talk zone, the $\mathrm{CR}$ user reduces the radius of the no-talk zone of the PU to the original value which is $R_{\text {no_talk }}$. Therefore, the sensing threshold will increase accordingly. After the CR user entered the revised notalk zone, the CR user performs spectrum sensing with period of $t_{s e n}^{\min }$. The procedure of the adaptive sensing period scheme is shown in Fig.4.

\section{Performance Evaluation}

We now compare the performance of SARS that we have considered in this paper in the various environments. In the performance evaluation, we assumed the multi-band CR environment in which the PU is the DTV station [7] and the number of CR bands is 60 . We let the radius of the no-talk zone of the PU be $480 \mathrm{~km}$ by assuming that the transmission power of the PU is $1 \mathrm{MW}$ and the antenna height is $60 \mathrm{~m} \mathrm{[7].}$ And we also let the path loss exponent, $\alpha$, be 3.8 and the path loss constant, $\kappa$ was set so that the received signal power at

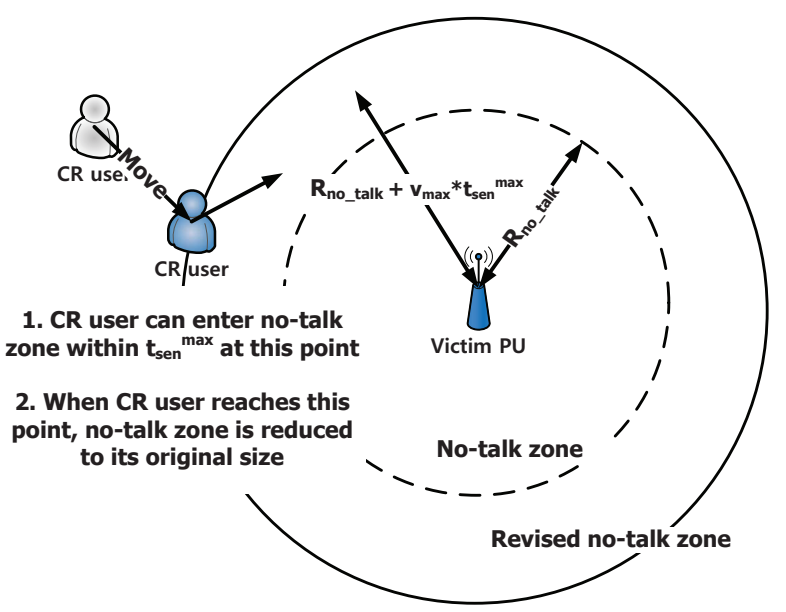

Fig. 4. Procedure of an adaptive sensing period scheme

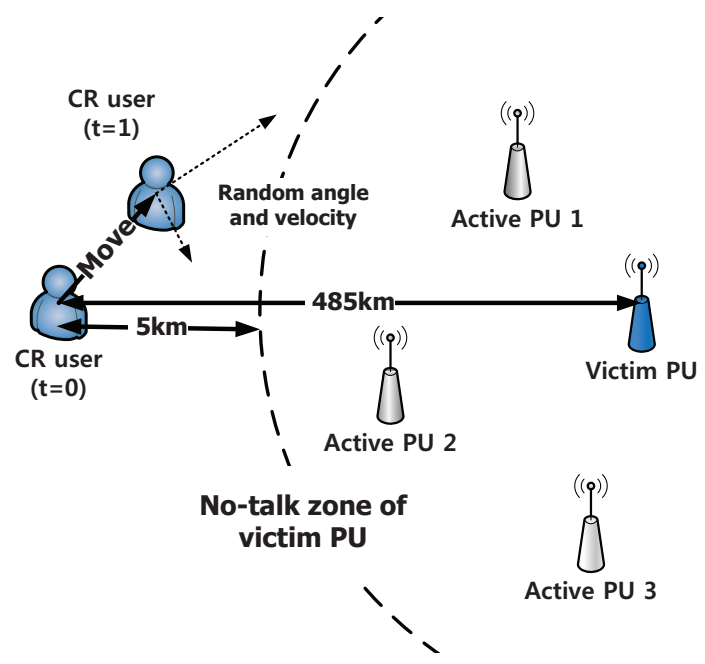

Fig. 5. Simulation environments

the edge of no-talk zone is $-114 \mathrm{dBm}$. Moreover, we assumed that $N 0$ is $-174 \mathrm{dBm} / \mathrm{Hz}$ and the bandwidth of the $\mathrm{PU}$ is $6 \mathrm{MHz}$ [10] [11]. For energy detection, we assumed that the sampling frequency is the same as the bandwidth of the PU [12].

In the performance evaluation, we assumed that a CR user is initially located $5 \mathrm{~km}$ away from the no-talk zone of a victim PU which uses the same band with the CR user. The CR user moves with random velocity and angle, and the distribution of velocity and angle is uniform distribution. The velocity of the CR user is randomly chosen from the range $\left[0, v_{\max }\right]$ and the angle of the velocity is randomly chosen from the range $\left[-\frac{\pi}{2}, \frac{\pi}{2}\right]^{7}$. We also let the CR user changes its velocity and the angle of movement in every 2 seconds. The simulation environments are shown in Fig.5.

First, we considered a DB based scheme and compared the performance of a GPS assisted SARS and a spectrum sensing assisted SARS with different $P_{M D}^{t h r}$. We used the number of GPS readings, which is the number of location update using

\footnotetext{
${ }^{7}$ We used $\left[-\frac{\pi}{2}, \frac{\pi}{2}\right]$ for the distribution of heading angle to make the CR user moves toward the victim PU.
} 


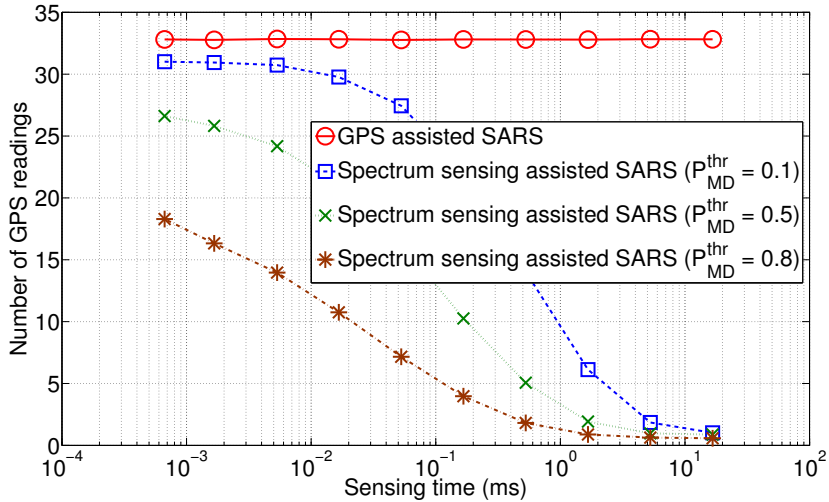

Fig. 6. Number of GPS readings vs. sensing time in DB based scheme

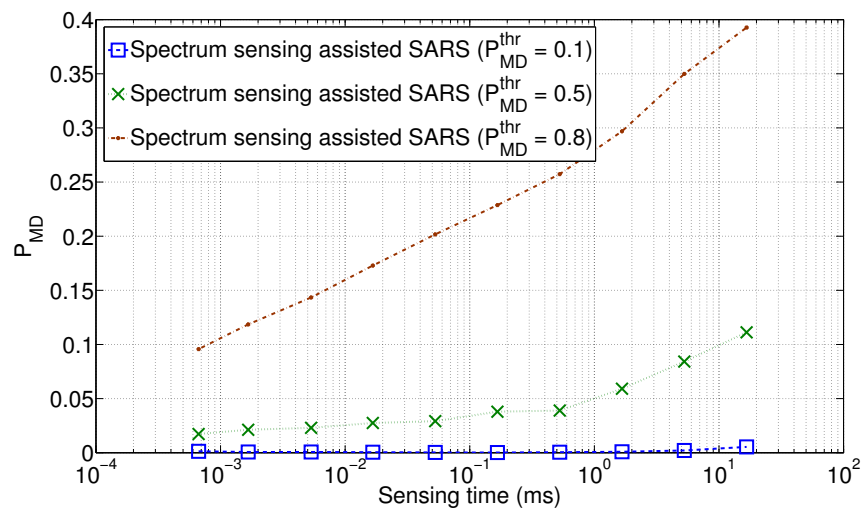

Fig. 7. $P_{M D}$ vs. sensing time in DB based scheme

GPS, and the $P_{M D}$, which is the probability that the victim $\mathrm{PU}$ is interfered by a CR user, as the performance metric. Given that the $P_{M D}$ of GPS assisted SARS will be zero, the GPS assisted SARS is not shown when $P_{M D}$ is considered. In the performance evaluation, we assumed that $t_{\text {min }}^{G P S \text { update }}$, which is the minimum duration between two consecutive GPS readings, is 2 seconds. First, we calculated the number of GPS readings and $P_{M D}$ by varying the sensing time. In the simulation, we let $v_{\max }=30 \mathrm{~km} / \mathrm{h}$. The simulation results are shown in Fig.6 and Fig.7.

As we can see from Fig.6, the number of GPS readings can be significantly reduced by using a spectrum sensing based SARS. We can also find that the number of GPS readings can be further reduced by increasing $P_{M D}^{t h r}$, because when $P_{M D}^{t h r}$ is increased, $\epsilon_{d}$ in a spectrum sensing assisted SARS will increase and the CR user will be more reluctant to use GPS. Although the number of GPS readings can be reduced by using high $P_{M D}^{t h r}, P_{M D}$ will increase in this case, as we can see from Fig.7.

We can also find that $P_{M D} \leq P_{M D}^{t h r}$ in all ranges, because the CR user performs multiple spectrum sensing before it reaches to the no-talk zone of a victim PU. Given that the CR user will perform multiple spectrum sensing near the notalk zone, the condition, $T\left(y_{d_{\text {curr }}}\right)-T\left(y_{d_{\text {init }}}\right)>\epsilon_{d}$ is likely

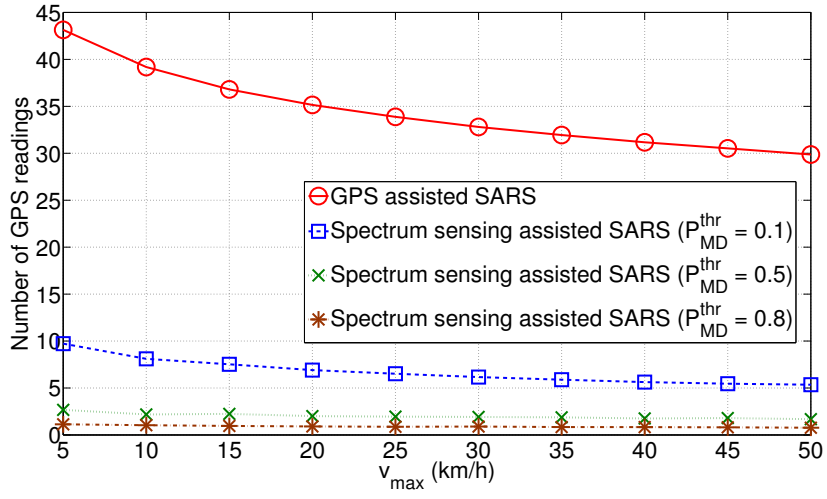

Fig. 8. Number of GPS readings vs. $v_{\max }$ in DB based scheme

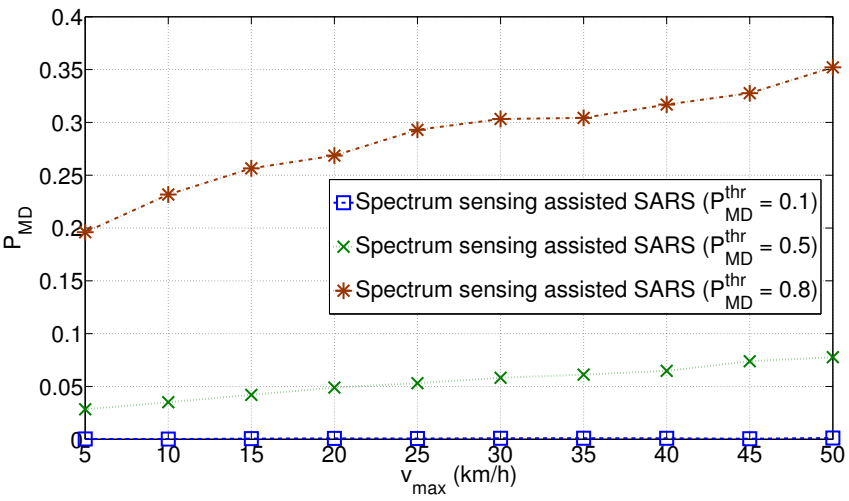

Fig. 9. $P_{M D}$ vs. $v_{\max }$ in DB based scheme

to be satisfied in at least one sensing attempts. And given that the CR user starts to use GPS after this condition is satisfied, the $P_{M D}$ is less than $P_{M D}^{t h r}$.

Moreover, we can also find that the number of GPS readings can be significantly reduced even when sensing time is short. This is due to the fact that we used a test PU which is close to a CR user. Therefore, the energy and time spent for the sensing which is used in the spectrum sensing assisted SARS will be small and it shows the validity of the scheme. We also find that the number of GPS readings decreases and $P_{M D}$ increases as sensing time increases, because the accuracy of energy detection increases. When the sensing time is short, $T\left(y_{d_{\text {curr }}}\right)$ will have high variance and the condition $T\left(y_{d_{\text {curr }}}\right)-T\left(y_{d_{\text {init }}}\right)>\epsilon_{d}$ is likely to be satisfied even when the CR user is not close to the no-talk zone.

Next, we calculated the number of GPS readings and $P_{M D}$ by varying $v_{\max }$. In the simulation, we let sensing time be $1.67 \mathrm{~ms}$. The simulation results are shown in Fig.8 and Fig.9.

As we can see from Fig.8, the number of GPS readings can be significantly reduced by using a spectrum sensing based SARS, even when the sensing time is short. We can also find that the number of GPS readings decreases as $v_{\max }$ increases, because the $\mathrm{CR}$ user can enter the no-talk zone of a victim PU more faster. Moreover, we can see from Fig.9 that $P_{M D}$ decreases as $v_{\max }$ increases, because the number of sensing 


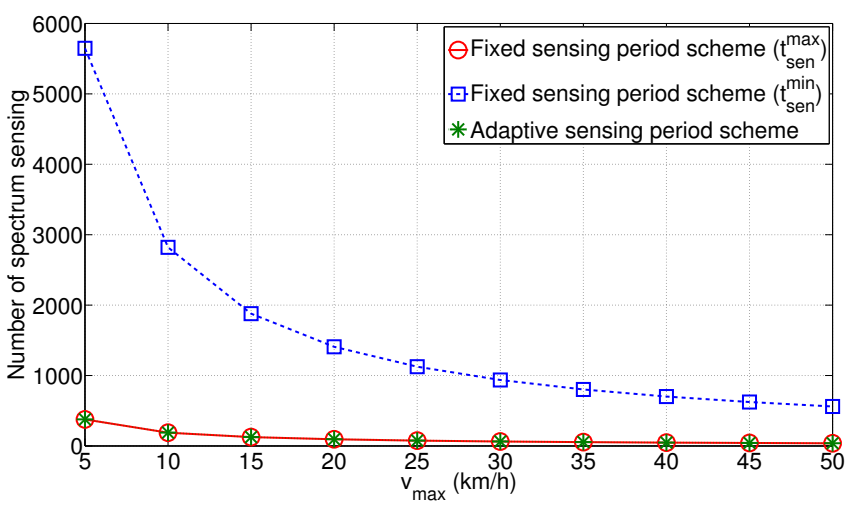

Fig. 10. Number of spectrum sensing attempts vs. $v_{\max }$ in sensing based scheme

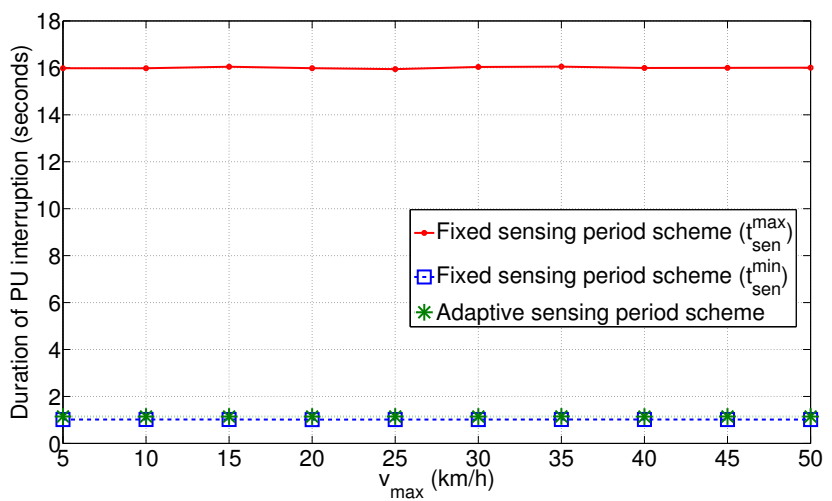

Fig. 11. Duration of PU interruption vs. $v_{\max }$ in sensing based scheme

attempts before the CR user enters the no-talk zone decreases. Given that the number of sensing attempts decreases, the probability that the condition $T\left(y_{d_{\text {curr }}}\right)-T\left(y_{d_{\text {init }}}\right)>\epsilon_{d}$ is satisfied before the CR user enters the no-talk zone, decreases.

Next, we considered a sensing based scheme. In this simulation, we compared the performance of a fixed sensing period scheme and an adaptive sensing period scheme. In the fixed sensing period scheme, the period of spectrum sensing is fixed to $t_{s e n}^{\max }$ or $t_{s e n}^{\min }$. And we assumed that $t_{s e n}^{\max }$ and $t_{s e n}^{\min }$ are 30 seconds and 2 seconds, respectively [9] [10]. In the simulation, we used the number of spectrum sensing attempts, which is the number of sensing attempts before the $\mathrm{CR}$ user enters the no-talk zone of a PU, and the duration of PU interruption, which is the time that the PU is interfered by the CR user, as the performance metric. The simulation results are shown in Fig.10 and Fig.11.

As we can see from the figures, the duration of PU interruption of an adaptive sensing period scheme is almost the same as that of a fixed sensing period scheme with maximum sensing period while the number of sensing of the adaptive sensing period scheme is almost the same as that of the fixed sensing period scheme with minimum sensing period. Therefore, by using the adaptive sensing period scheme, the number of sensing can be maintained low while the interference to a
PU can be minimized. We can also find that the number of the spectrum sensing decreases as $v_{\max }$ increases, because the $\mathrm{CR}$ user can enter the no-talk zone of the PU more faster.

\section{CONCLUSION}

Herein, we consider the change of spectrum availability which is caused by the mobility of a CR user, and propose a SARS for both CR systems with a DB based scheme and that with a sensing based scheme. In the SARS for CR systems with the DB based scheme, the period of location update is changed according to the velocity of $\mathrm{CR}$ users and the spectrum sensing can be used to reduce the overhead of the SARS. In the SARS for CR systems with the sensing based scheme, the period of spectrum sensing changes according to the velocity of CR users. Through the analysis and the simulation results, we have shown that the spectrum availability of the CR users can be efficiently checked by using the proposed SARS, when the CR user moves. We also have shown that the overhead which is caused by our SARS is low.

\section{ACKNOWLEDGEMENT}

This research was supported by the KCC (Korea Communications Commission), Korea, Under the R\&D program supervised by the KCA (Korea Communications Agency) (KCA-2011-08913-04001). This work was also supported by Brain Korea 21 Project, BK Electronics and Communications Technology Division, KAIST in 2012.

\section{REFERENCES}

[1] J. Mitola, et al., "Cognitive radio: Making software radios more personal", IEEE Pers. Commun., vol. 6, no. 4, pp. 13 - 18, Aug. 1999.

[2] D. Cabric, A. Tkachenko, and R. W. Brodersen, "Experimental study of spectrum sensing based on energy detection and network cooperation," ACM 1st Intl. Workshop on Technology and Policy for Accessing Spectrum (TAPAS), p.12-es, Aug. 2006.

[3] W. Jianfeng, M. Ghosh, and K. Challapali, "Emerging cognitive radio applications: A survey," IEEE Commun. Mag., vol. 49, no. 3, pp. 74-81, Mar. 2011.

[4] S. Yin, D. Chen, Q. Zhang, M. Liu, and S. Li, "Mining Spectrum Usage Data: a Large-Scale Spectrum Measurement Study," MobiCom'09,pp. 13-24, 2009.

[5] W. Lee, and D.H. Cho, "Cooperation based spectrum management scheme in ad-hoc cognitive radio system ," IEEE Crowncom 2011, pp.36-40, Jun. 2011.

[6] W. Lee, and D.H. Cho," "Comparison of Channel Information Acquisition Schemes in Cognitive Radio System," to be appeared in IEEE WCNC 2012

[7] R. Murty, R. Chandra, T. Moscibroda, and P. Bahl, "SenseLess: A database-driven white spaces network," IEEE DySPAN 2011, pp.10-21, May. 2011.

[8] W. Lee, and D.H. Cho, "Mean velocity estimation of mobile stations by spatial correlation of channels in cellular systems ," IEEE Communications Letters, vol.13, no.9, pp.670-672, Sept. 2009.

[9] INFSO-ICT-248303 QUASAR, Deliverable D1.2 Regulatory feasibility assessment, Dec. 2010, [Online]. Available: http://www.quasarspectrum.eu/downloads/publicdeleverables. html.

[10] W. Lee, and D.H. Cho, "Enhanced spectrum sensing scheme in cognitive radio systems with MIMO antennae," IEEE Trans. Veh Tech., vol.60, no.3, pp.1072-1085, Mar. 2011.

[11] W. Lee, and D.H. Cho, "Distributed scheduling algorithm for cooperative transmission with multiple relays," IEEE Crowncom 2011, pp.31-35, Jun. 2011.

[12] E. Peh, Y.-C. Liang, Y.L. Guan, and Y. Zeng, "Optimization of cooperative sensing in cognitive radio networks: a sensing-throughput tradeoff view," IEEE Trans. Wireless Comm., vol.58, no.9, pp.5294-5299, Nov. 2009. 